\title{
Potential role of RING finger protein 166 (RNF166), a member of an ubiquitin ligase subfamily, involved in regulation of $T$ cell activation
}

\author{
PING YANG ${ }^{* 1,2}$, YILU LU $U^{*}$, XUE JIANG ${ }^{l}$, MINHUI LI $I^{3}$, CHAO LI $I^{l}$, HUIJUAN CHEN ${ }^{l}$, KUN ZHANG ${ }^{l}$, \\ KEJIAN PAN ${ }^{2}$ DACHANG TAO ${ }^{l}$, SIZHONG ZHANG ${ }^{l}$, YONGXIN MA ${ }^{l}$
}

\author{
${ }^{1}$ Department of Medical Genetics \& Division of Morbid Genomics, State Key Laboratory of Biotherapy, West China Hospital, Sichuan \\ University, Chengdu, P.R. China \\ ${ }^{2}$ Department of Biomedicine, Chengdu Medical College, Chengdu, P.R. China \\ ${ }^{3}$ Center of Science and Research, Chengdu Medical College, Chengdu, P.R. China
}

*These authors contributed equally to this work.

\begin{abstract}
RING (really interesting new gene) finger protein 166, or RNF166, belongs to a C3HC4 ubiquitin ligases subfamily, which include four related proteins containing a conserved C3HC4 (Cys3-His-Cys4) RING finger domain. RNF125, one member of the subfamily, has been identified as a regulator of $T$ cell activation, but the potential roles of another member RNF166 remains poorly understood. Here we reported that RNF166 is involved in regulation of T cell activation. Flow cytometry (FCM) data showed that overexpression of RNF166 in primary $T$ cells and Jurkat $T$ cells induced over 2-fold increase of CD69, a T-cell activation marker, suggesting that RNF166 is a positive-regulator of T cell activation. Furthermore, pull-down assays showed that RNF166 can bind with both Lys ${ }^{48}$-linked polyubiquitin and Lys ${ }^{63}$-linked polyubiquitin, indicating that RNF166 may play regulating roles in $T$ cell activation by selfdegradation via ubiquitin-proteasome pathway and/or cross-talk with certain signaling pathways via non-proteasome-dependent pathways. In conclusion, our work reveals that RNF166 is a potential positive-regulator of $T$ cell activation and these findings provide a novel insight into understanding the functions of RNF166 in the positive regulation of immune responses.
\end{abstract}

Key words: flow cytometry, pull down, regulator, RING finger protein 166, T cell activation.

(Centr Eur J Immunol 2013; 38 (1): 15-22)

\section{Introduction}

Ubiquitination plays crucial roles in modulation of $\mathrm{T}$ cells functions by attaching monoubiquitin or polyubiquitin to various target proteins, such as nuclear factor $\kappa \mathrm{B}$ $(\mathrm{NF}-\kappa \mathrm{B})$, extracellular signal regulated kinase (ERK) 1/2, protein kinase $\mathrm{C} \theta$ (PKC $\theta)$, phospholipase $\mathrm{C} \gamma(\mathrm{PLC} \gamma)$ and $70 \mathrm{kDa} \zeta$ chain associated protein kinase (ZAP-70) [1-4]. It is well known that E3 ubiquitin ligases can selectively recognize target substrate-proteins and interact with E2 ubiquitin conjugating enzymes via E2 interacting domains, e.g. RING (really interesting new gene) domain [5].
Recently, a RING domain protein RNF125 (alias T cell RING protein in activation 1, TRAC-1), which predominantly expressed in lymphoid cells, was identified as a novel positive regulator of $\mathrm{T}$ cells activation among ubiquitin ligases in a functional screen for T cells regulators [6, 7]. Homologous protein BLAST searched with RNF125 reveals that this protein belongs to a subfamily of E3 ubiquitin ligases with zinc fingers, including RNF114 (alias ZFP313), RNF138 (alias NLK-associated RING finger protein, NARF) and RNF166 [6]. All members of this subfamily comprise five highly conserved domains, including

Correspondence: Yongxin Ma, $1^{\text {st }}$ Keyuan 4 Lu, GaopengDadao, High-Tech Zone, Chengdu, China 610041, tel. 86-28-85164010, fax 86-28-85164009, e-mail: mayongxin@gmail.com 
an amino-terminal C3HC4 (Cys3-His-Cys4) RING finger domain, a central C2HC (Cys2-His-Cys), two C2H2 (Cys2His2)-type zinc fingers and a carboxy-terminal UIM (ubiquitin interacting motif) domain. The structural similarity among these family members suggests that these proteins may have similar functions in cell physiology. The membrane-bound protein RNF125 has been identified as a positive-mediator of T cells activation; RNF114 has been characterized as a psoriasis susceptibility gene; and RNF138 has been confirmed as an inhibitor of Wnt signaling pathway. However, the roles of RNF166 remain poorly described, especially in immune responses [8]. Here we present that RNF166 may play a potential role in positively regulating of $\mathrm{T}$ cells activation, providing new insights into understanding the functions of RNF166.

\section{Material and methods}

\section{Cells preparation and culture}

Primary human $\mathrm{T}$ cells were enriched from human peripheral blood mononuclear cells (PBMCs) by the depletion of indirectly magnetically labeled non T cells using Pan T cell Isolation Kit II (Miltenyi Biotec, Germany). Normally, more than $90 \%$ pure T cell populations were isolated, measured by flow cytometry analysis with PE conjugated mouse monoclonal anti-human $\mathrm{CD} 3 \mathrm{e}$ antibody using a FACScalibur (Becton Dickinson, CA, USA). Purified T cells were maintained in RPMI 1640 medium plus $10 \%$ FCS, 100 units $/ \mathrm{ml}$ penicillin and $0.1 \mathrm{mg} / \mathrm{ml}$ streptomycin at $37^{\circ}$ under $5 \% \mathrm{CO}_{2}$ and must be used for the subsequent experiments within $24 \mathrm{~h}$. The human peripheral blood samples were obtained from healthy adult volunteers in West China First University Hospital. The study was approved by the committee of Ethics of Biological and Medical Research, Sichuan University, and all the participants gave written informed consent for scientific research. Human Jurkat T cells (Clone E6-1, ATCC) were maintained in RPMI 1640 medium supplemented with $10 \%$ FCS, 100 units $/ \mathrm{ml}$ penicillin and $0.1 \mathrm{mg} / \mathrm{ml}$ streptomycin at $37^{\circ}$ under $5 \% \mathrm{CO}_{2}$. All following experiments were repeated at least three times unless stated otherwise.

\section{cDNAs and constructs}

Total RNA was extracted from prepared Jurkat T cells with Trizol reagent (Invitrogen, USA) according to manufacturer's protocol. The coding sequence of RNF166 (GenBank accession number: BC013948.2) was amplified by RT-PCR and cloned into pEGFP-C1 (Clontech, USA) and pGEX-5X3 (GE Healthcare Life Sciences, Germany) vectors to express proteins with $N$-terminal GFP- or GST-tag respectively. The sequences of the PCR primers (with restrict enzyme site) used are as follows: RNF166-F: 5'-CCC AAG CTT GGA TGG CTA TGT TCC GC-3'; RNF166-R: 5'-CGC GGA TCC CTG CGC TTC CCT
TCA GTT CTC AG-3'. The truncated mutant proteins were obtained by segmented PCR and fusion PCR and cloned into pEGFP-C1 vectors. The sequences of the PCR primers used are as follows: RNF166- $\triangle$ RING-F: 5'-GAG GCG CAG TAC ACC CGC CTG CCC TTC GAC-3'; RNF166$\triangle$ RING-R: 5'-TTG GGG TCG AAG GGC AGG CGG GTG TAC TGC GCC TCC AGG C-3'; RNF166- $\triangle$ C2HC-F: 5'-TCC TAC AAA GCG CCC CTG AAG GTC CAG GAG-3'; RNF166- $\triangle$ C2HC-R: 5'-CTC CTG GAC CTT CAG GGG CGC TTT GTA GGA-3'; RNF166- $\Delta$ C2H2-IF: 5'-AGG TCC ACC TTC GCC CGC AGC GAC CCC AAC-3'; RNF166- $\triangle$ C2H2-I-R: 5'-GTT GGG GTC GCT GCG GGC GAA GGT GGA CCT-3'; RNF166- $\Delta$ C2H2-II-F: 5'-TGG GGG GAC CCC AGC AAG TTC TCC TAC GAC-3'; RNF166- $\triangle$ C2H2-II-R: 5'-GTC GTA GGA GAA CTT GCT GGG GTC CCC CCA-3'; RNF166- $\triangle$ UIM-R: 5'-CGC GGA TCC CAC AAA GGT GTC GTA G-3'. All RNF166 constructs were verified by sequencing.

\section{Western blot analysis}

Harvested cells were lysed in ice-cold universal protein extraction buffer (Bioteke, Beijing) supplemented with protease inhibitor cocktail (Roche, USA) for $30 \mathrm{~min}$. Cell lysates were separated on 4-12\% SDS-page gel and transferred to a polyvinylidene fluoride (PVDF) membrane. Membranes were blocked in TBS-T Buffer (50 mM Tris$\mathrm{HCl}, 150 \mathrm{mM} \mathrm{NaCl}, 0.1 \%$ Tween-20, PH 7.6) supplemented with $5 \%$ nonfat dry milk. The membranes were incubated overnight at $4{ }^{\circ} \mathrm{C}$ with the indicated primary antibody: rabbit polyclonal anti-GFP antibody (eBioscience, USA); rabbit polyclonal anti-human RNF166 antibody (Abcam, USA) and rabbit polyclonal anti-human beta actin antibody (AVIVA Systems Biology, USA), followed by $5 \mathrm{~min}$ washes in TBS-T for three times and incubation with HRPlabeled secondary antibody (Zhongshan Goldenbridge, China) in TBS-T for 1 hour at RT. The membranes were detected with chemiluminescent HRP substrate (Millipore, USA). using a ChemiDoc XRS System (Bio-Rad, CA, USA).

\section{Transfection and transformation}

For transfection, $10^{7}$ Jurkat T cells and $5 \times 10^{6}$ primary $\mathrm{T}$ cells were electroporated in $250 \mu \mathrm{l}$ of OPTI-MEM I Reduced Serum medium without FCS and antibiotics to produce RNF166-GFP and truncated-RNF166-GFP fusion proteins, using $0.2 \mathrm{~cm}$ cuvettes and Gene Pulser Xcell II Electroporation System (Bio-Rad, CA, USA) at $180 \mathrm{~V}$ and $1000 \mu \mathrm{F}$ in the presence of $20 \mu \mathrm{g}$ of recombinant DNA. Untransfected T cells and wild-type GFP transfected T cells were employed as negative controls. For transformation, Escherichia coli strain BL21 (DE3) cells were electroporated with RNF166 constructs cloned into pGEX-5X3 to allow production of RNF166-GST fusion proteins at 1800 $\mathrm{V}, 25 \mu \mathrm{F}$ and $200 \Omega$ using Gene Pulser Xcell II Electropo- 
ration System in $0.1 \mathrm{~cm}$ cuvettes (Bio-Rad, CA, USA). The negative controls were untransformed Escherichia colis and wild-type GST transformed Escherichia colis.

\section{$T$ cells activation and flow cytometry analysis}

$8 \times 10^{4}$ transfected primary human T cells or Jurkat T cells cultured in a 96-well plate were stimulated by Dynabeads Human T-Activator CD3/CD28 (Invitrogen Dynal AS, Norway) with a bead-to-cell ratio of $1: 1$ for $16 \mathrm{~h}$ at $37^{\circ} \mathrm{C}$, followed with $30 \mathrm{~min}$ of incubation with APC-conjugated mouse monoclonal anti-human CD69 antibody (eBioscience, USA) on ice in darkness. Subsequently, cells were fixed in $4 \%$ paraformaldehyde and counted by flow cytometry using FACScalibur (Becton Dickinson, USA) gated on healthy cells based on forward and side scatter properties. Instrument settings were optimized by transfected but unstained $\mathrm{GFP}^{+}$and APC-CD69-stained GFP cells. Analysis was carried out by FCS Express Version IV software (De Novo, USA) and CellQuest (Becton Dickinson, USA).

\section{Polyubiquitin pull-down assay}

GST-RNF166 fusion proteins were purified from $5 \mathrm{ml}$ Escherichia coli strain BL21 cultures on glutathione Sepharose 4 fast flow kit (GE Healthcare, Germany) and immobilized on the Sepharose beads according to the manufacture's protocol. $1 \mathrm{ml}$ GST-RNF-beads and control GSTbeads were respectively incubated with $1 \mu \mathrm{g}$ Lys ${ }^{48}$-linked or Lys ${ }^{63}$-linked polyubiquitin 2-7 chains overnight at $4{ }^{\circ} \mathrm{C}$. The protein/bead mixtures were washed in PBS for three times, then denatured at $95^{\circ} \mathrm{C}$ for 5 min and loaded onto $10 \%$ SDS-polyacrylamide gels. Blots were subjected to immunoblotting as above. Ubiquitin pulldown was verified with mouse monoclonal anti-human ubiquitin antibody (Covance, Princeton, USA) and goat polyclonal anti-GST antibody (GE Healthcare, Germany).

\section{Results}

\section{Endogenous expression of RNF166 protein in T lymphoid cells}

Previous reports described that human RNF166 has three transcript variants, which putatively encode 237 aa, 156 aa and 128 aa peptides respectively $[9,10]$. To verify which of the isoforms expressed in T cells, we performed a western blot using the rabbit polyclonal anti-human RNF166 antibody which recognizes the co-owned $C$-terminal sites of these isoforms (Fig. 1A). Western blot analysis showed that only the longest isoform $(26.1 \mathrm{kD})$ was detected in primary T cells, as well as Jurkat T cells. The encoded protein contains intact $N$-terminal RING, C2HC, two $\mathrm{C} 2 \mathrm{H} 2$ and $C$-terminal UIM domains. This result also confirmed that RNF166 is endogenously expressed in T lymphoid cells (Fig. 1B).

\section{Homologous blast and constructs of RNF166 protein}

Protein BLAST searches with RNF166 amino acid sequence reveal that it belongs to a subfamily of small C3HC4 RING ubiquitin ligases which contain C3HC4 (Cys3-His-Cys4) RING finger domain [6]. The homologous sequences are present in other three human proteins: RNF114, RNF138 and RNF125. Five conserved domains: C3HC4 RING domain (33-72 aa), C2HC domain (98-117 aa), C2H2-I domain (152 -173 aa), C2H2-II domain (182-208 aa) and UIM domain (222 aa-236 aa), were respectively located on RNF166 protein from $N$-terminus to $C$-terminus (Fig. 2A). To study the function of RNF166, vectors laboring $N$-terminal GFP- or GST-tagged RNF166 recombinants (pEGFP-RNF166, pGEX-RNF166), as well as five GFPtagged RNF166 domain-deleted mutants (pEGFP-RNF166$\triangle$ RING, pEGFP-RNF166- $\triangle$ C2HC, pEGFP-RNF166$\Delta$ C2H2-I, pEGFP-RNF166- $\Delta$ C2H2-II and pEGFP-RNF166$\triangle \mathrm{UIM}$ ) were constructed and identified with PCR methods (Fig. 2B and 2C).

\section{Identification of RNF166 as a potential positive regulator of $T$ cells activation}

To investigate whether RNF166 plays roles as a regulator of $\mathrm{T}$ cells activation induced by CD3/CD28 double signals, a series of constructs had been generated as described above. Primary human T cells were isolated with Pan T cell Isolation Kit II from PBMCs and analyzed by anti-CD3e-PE using FACScalibur. The results showed that 98.2\% pure $\mathrm{T}$ cells populations were isolated (Fig. 3B). pEGFP-C1, pEGFP-RNF166 recombinant vectors and the domain-deletion mutants were separately transfected into primary human $\mathrm{T}$ cells and Jurkat $\mathrm{T}$ cells by electroporation. The transfected $\mathrm{T}$ cells were stimulated with Dynabeads Human T-Activator CD3/CD28 (Fig. 3A) and incubated with APC conjugated mouse monoclonal anti-human CD69 antibody to detect the expression level of CD69, a early marker of T-cell activation, by flow cytometry (Fig. 3C and 3E).

As shown in Fig. 3C and 3E, the left quadrants dots $\left(\mathrm{GFP}^{-}\right)$indicated the $\mathrm{T}$ cells with RNF166 endogenous expression, the right quadrants dots $\left(\mathrm{GFP}^{+}\right)$indicated the $\mathrm{T}$ cells in which RNF166 or its mutants were overexpressed, the lower quadrants dots $\left(\mathrm{CD} 69^{-}\right)$indicated the T cells which were not activated, the upper quadrants dots $\left(\mathrm{CD} 69^{+}\right)$ indicated the T cells which were successfully activated. The ratio of upperleft quadrant dots against the upperleft plus lowerleft quadrants dots $\left[\mathrm{CD}^{+} 9^{+}\left(\mathrm{GFP}^{-}\right)\right]$indicated the percentage of $\mathrm{CD}^{2} 9^{+}$cells in the $\mathrm{GFP}^{-}$cells, and the ratio of upperright quadrant dots against the upperright plus lowerright quadrants dots $\left[\mathrm{CD} 9^{+}\left(\mathrm{GFP}^{+}\right)\right]$indicated the percentage of $\mathrm{CD} 9^{+}$cells in the $\mathrm{GFP}^{+}$cells. The ratio of $\mathrm{CD}^{2} 9^{+}\left(\mathrm{GFP}^{+}\right)$against $\mathrm{CD}^{+} 9^{+}\left(\mathrm{GFP}^{-}\right)$indicated the efficiency changes of $\mathrm{T}$ cells activation. 
$\mathrm{C} 2 \mathrm{HC}$ domain

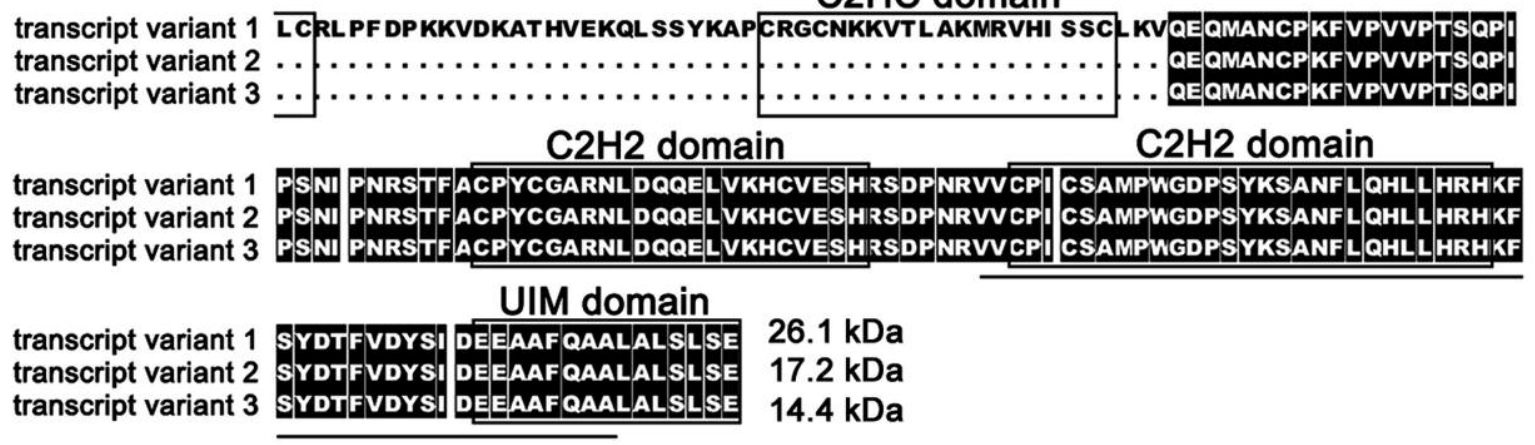

B

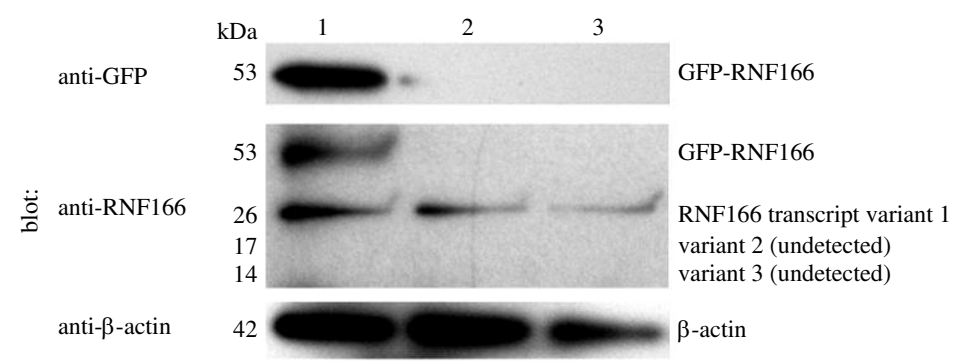

Fig. 1. Endogenous expression analysis of RNF166 isoform 1 in T lymphoid cells. A) Amino acid alignments of three isoforms of human RNF166 protein, encoded by transcript variant 1 (NM_178841.3), transcript variant 2 (NM_001171815.1) and transcript variant 3 (NM_001171816.1) respectively. Alignments were performed using DNAMAN software (Lynnon, Quebec, Canada). Identical residues are highlighted in black. Block symbols indicate the domains of RNF166. Underlines indicate the co-owned recognized sites of the polyclonal antibody. B) Isoform 1 (26.1 kDa) of RNF166 protein was detected both in primary $\mathrm{T}$ cells and Jurkat $\mathrm{T}$ cells by western blot analysis, confirming that RNF166 can be endogenously expressed in T lymphoid cells (lane: 1. Jurkat T cells transfected with pEGFP-RNF166; 2. untransfected Jurkat T cells; 3. untransfected primary T cells); antiGFP and anti- $\beta$-actin antibodies were employed as controls

Flow cytometry analysis by calculating the ratio of $\mathrm{CD} 9^{+}\left(\mathrm{GFP}^{+}\right)$against $\mathrm{CD}^{+} 9^{+}\left(\mathrm{GFP}^{-}\right)$in $\mathrm{GFP}^{+}$-total (fluorescence intensity $>10$ ), $\mathrm{GFP}^{+}$-low (fluorescence intensity 10-100) and $\mathrm{GFP}^{+}$-high (fluorescence intensity > 100) primary human T cells showed that CD69 expression was significant higher in RNF166 transfected $\mathrm{GFP}^{+}$cells compared with untransfected $\mathrm{GFP}^{+}$cells (ratio value 2.4 against 1.0 ), suggesting that RNF166 may be a potential positive regulator of $\mathrm{T}$ cells activation (Fig. 3D). The similar results were also found in Jurkat T cells (ratio values were 2.1 against 1.4) (Fig. 3F, left two panels). Interestingly, RING domain-deletion mutant, RNF166- $\Delta$ RING, could induce a large extent to upregulate the efficiency of $\mathrm{T}$ cells activation compared with the other four mutants (ratio valus were 5.5, 2.9, 3.6, 3.5 and 3.8 respectively), suggesting that the $N$-terminus including RING domain may play a negative role despite the positive regulation function of intact RNF166 protein in $\mathrm{T}$ cells activation (Fig. 3F, right five panels).

\section{Polyubiquitin pull-down analysis of recombinant GST-RNF166}

Homology blast indicates that RNF166 belongs to a RING-family with activity of E3 ubiquitin ligases contained an UIM domain. To confirm whether RNF166 is also involved in ubiquitination which induces itself degradation or signaling cross-talk with other non-proteasomedependent pathways by binding polyubiquitin, we performed polyubiquitin pull-down analysis by incubating GST-RNF166 fusion protein with free polyubiquitin chains. The results of western blotting performed with anti-ubiquitin and anti-GST antibodies, showed that RNF166 protein can efficiently pull down Lys $^{48}$ - and Lys ${ }^{63}$-linked polyubiquitin2-7 chains (Fig. 4). This result indicated that RNF166 protein may play regulating roles by ubiquitin-proteasome involved self-degradation via system (mediated by Lys $^{48}$-linked polyubiquitin chains) and/or play functions 


\begin{tabular}{|c|c|}
\hline $\begin{array}{l}\text { RNF114 } \\
\text { RNF166 } \\
\text { RNF138 } \\
\text { RNF125 }\end{array}$ & $\begin{array}{l}\ldots \text { MAAQQRDCGGAAQLAGPAAEADPLG. } \ldots \ldots \ldots \text { RF TPVCLEVYEKPVQVP. CGHVFCSACLQECLKP } \\
\text { MAMFRSLVASAQQRQPPAGPAGGDSGLEA. } \ldots \ldots \text { QYT CPI CLEVYHRPVAI GS CGHTFCGECLQPCLQV } \\
\ldots \ldots \ldots \ldots \ldots \text { MAEDLSAATSYTED. . . . DFY CPVCQEVKT VRTACQHVF CRKCFLTAMRE } \\
\ldots \text { MGSVLSTDSGKSAPASATARALERRRDPELPVTSF C CAVCLEVLHQPVRTR. CGHVFCRSCI ATSLKN }\end{array}$ \\
\hline & C2HC domain \\
\hline $\begin{array}{l}\text { NF166 } \\
\text { NF138 } \\
\text { NF125 }\end{array}$ & $\begin{array}{l}\text { PSPL CPLC LPF DPKK. . . VDKATHVEKQLSSYKAP CRGCNKKVTL AKMRVHI SS CI KVQE } \\
\text { SGAHCPL CR GNVTRRERACPERALDLENI MRKF SGS CRCCAKQI KFYRMRHHYKS CKKYQD } \\
\text { NKWTCPYCRAYLPSEG. . . . VPATDVAKRMKSEYKNGAECDTLVCLSEMRAHI RTCQKYI D }\end{array}$ \\
\hline
\end{tabular}

$$
\mathrm{C} 2 \mathrm{H} 2-\mathrm{I} \text { domain }
$$

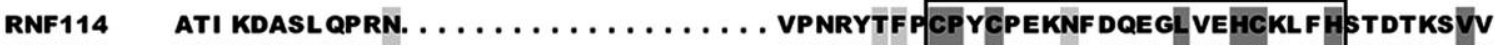
RNF166 PVVPTSQPI PSN. . . . . . . . . I PNRSTFA CPYCGARNL DQQEL VKHCVESHRSDPNRVV RNF138 PNFQI SQDSVGNSNRSETSTSDNTETYQENTSSSGHPTFKCPLCQESNFTRQRLLDHCNSNHLFQI VPVT RNF125

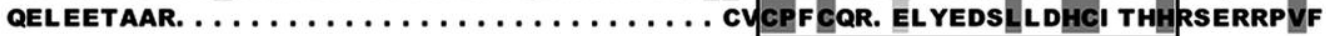

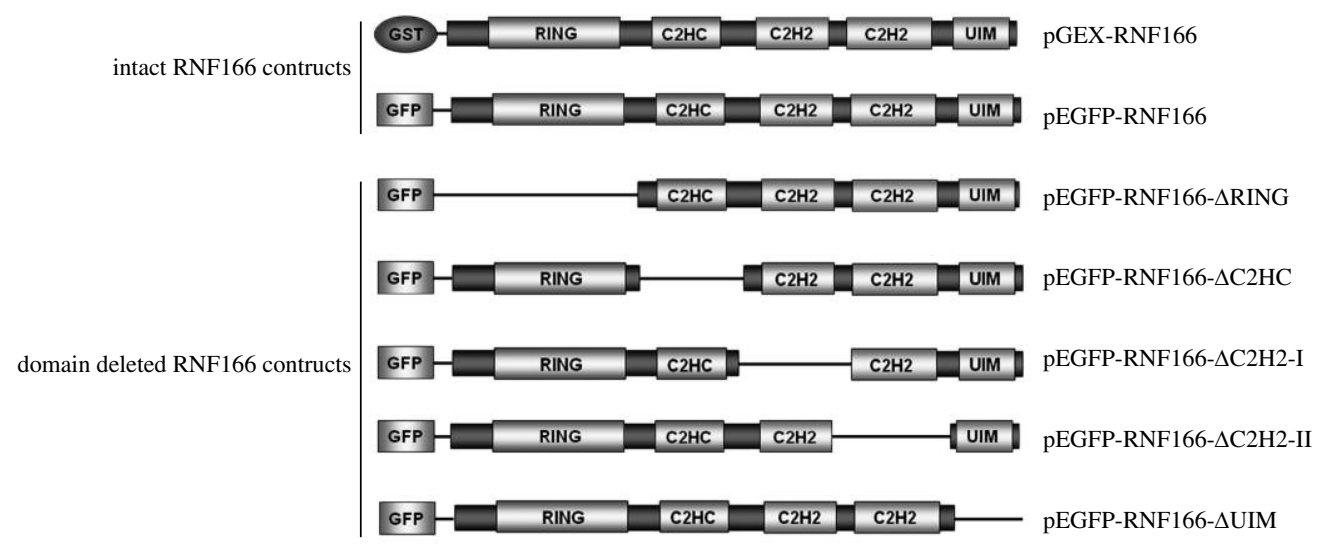

C

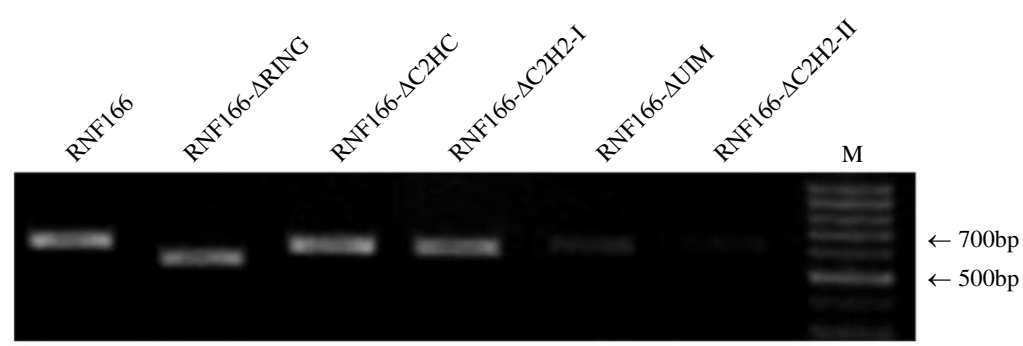

Fig. 2. RNF166 belongs to a RING ubiquitin ligases subfamily and the constructs built in this research. A) Amino acid alignments of the human proteins RNF166 (GenBank accession number Q96A37), RNF114 (GenBank accession number Q9Y508), RNF138 (GenBank accession number Q8WVD3) and RNF125 (GenBank accession number Q96EQ8). Alignments were performed using DNAMAN software (Lynnon Biosoft, Quebec, Canada). Identical residues are highlighted in darker grey, conservative changes are in light grey [6]. B) Schematic diagram of the intact and domain-deletion mutant constructs displayed the protein-tags (GST- or GFP-), the C3HC4 RING, C2HC, C2H2-I, C2H2-II and UIM domains. C) PCR products corresponding to B 
A

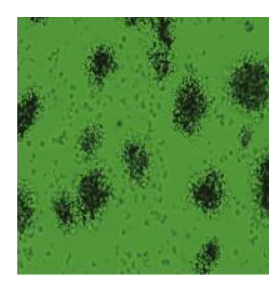

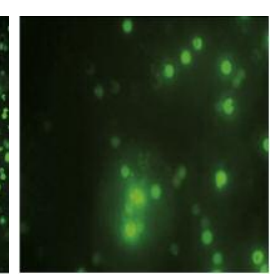

C

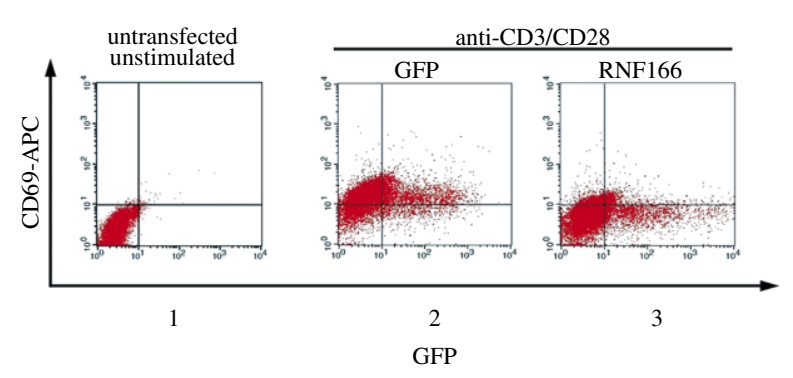

B

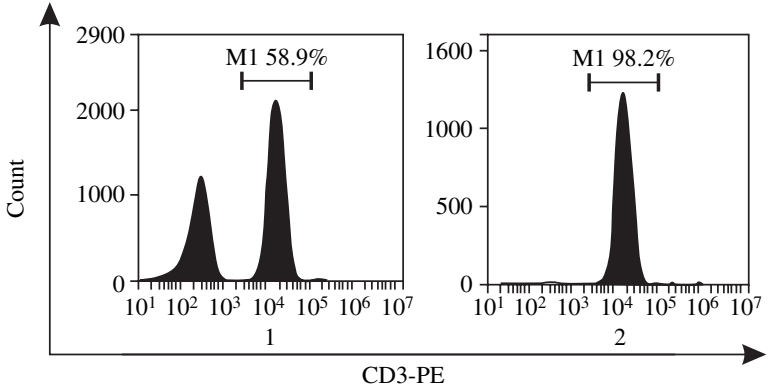

$\mathrm{D}$

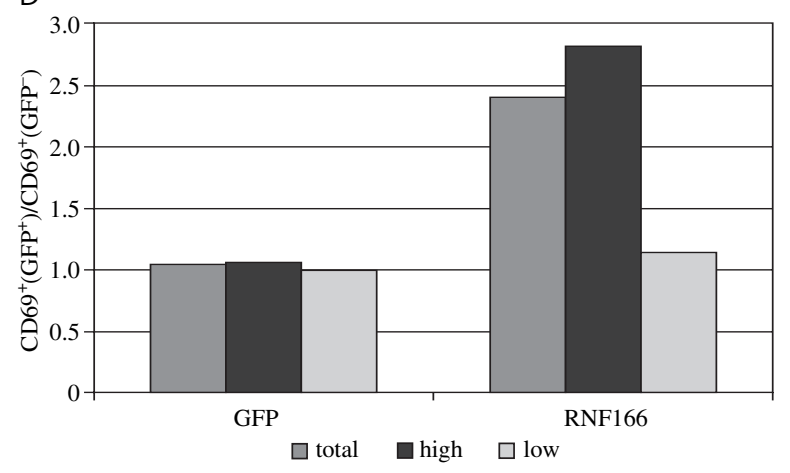

E

anti-CD3/CD28
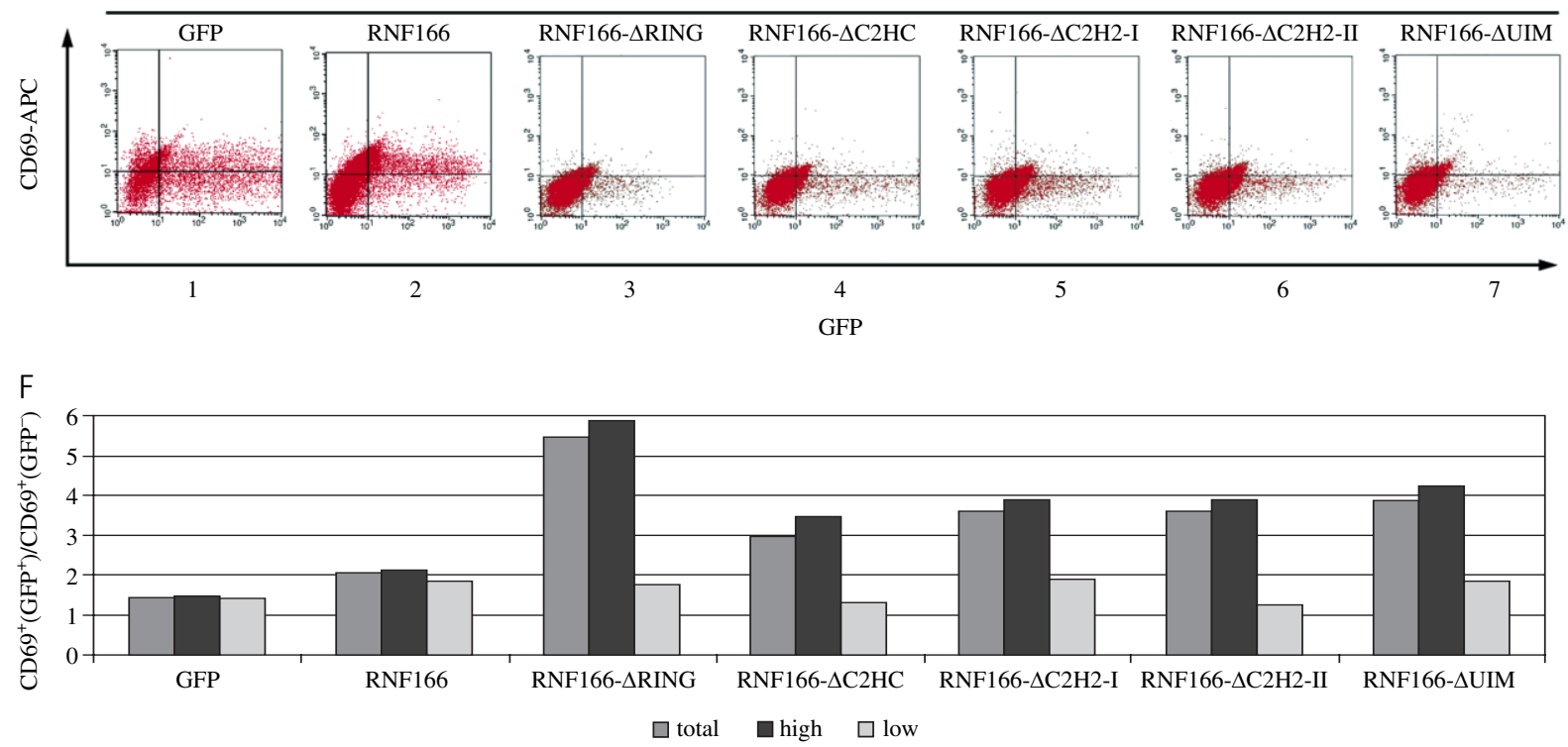

Fig. 3. RNF166 is a potential positive regulator of T cells activation. A) T cells were stimulated by Dynabeads Human T-Activator CD3/CD28. Left: green fluorescence indicates expression of GFP-RNF166; right: bright field. B) Human primary T cells were enriched from PBMCs by Pan T cell Isolation Kit II and analyzed by anti-CD3e-PE using FACScalibur. Left: pre-purification; right: post-purification. C) Activation of primary T cells (indicated by level of CD69, vertical axis) was analyzed by flow cytometry method. Left: Untransfected and unstimulated cells employed as control; middle: GFP-transfected cells stimulated as described before; right: stimulated cells transfected with GFP-tagged RNF166. D) The ratios of CD69+ $\left(\mathrm{GFP}^{+}\right)$against $\mathrm{CD}^{+} 9^{+}$ $\left(\mathrm{GFP}^{-}\right)$in primary T cells were calculated to evalue the efficiency changes of primary T cells activation separately for GFP+total (fluorescence intensity > 10), GFP+-low (fluorescence intensity 10-100) and GFP+-high (fluorescence intensity $>100$ ) populations. GFP: stimulated cells transfected with GFP; RNF166: stimulated cells transfected with GFP-tagged RNF166. E) Activation of stimulated Jurkat T cells (indicated by level of CD69, vertical axis) was analyzed by flow cytometry method. Panel 1: Jurkat T cells transfected with pEGFP-C1; Panel 2: Jurkat T cells transfected with pEGFP-RNF166; Panel 3-7: Jurkat T cells transfected with RNF166 domain-deleted mutants respectively; (3, RNF166- $\Delta$ RING; 4, RNF166- $\Delta$ C2HC; 5, RNF166$\Delta \mathrm{C} 2 \mathrm{H} 2-\mathrm{I} ; 6$, RNF166- $\Delta \mathrm{C} 2 \mathrm{H} 2-\mathrm{II} ; 7$, RNF166- $\Delta \mathrm{UIM}) \mathrm{F}$ ) Activation efficiencies (indicated by ratios of CD69+ $\left[\mathrm{GFP}^{+}\right]$against $\left.\mathrm{CD}^{+} 9^{+}\left[\mathrm{GFP}^{-}\right]\right)$in stimulated Jurkat T cells described above 
A
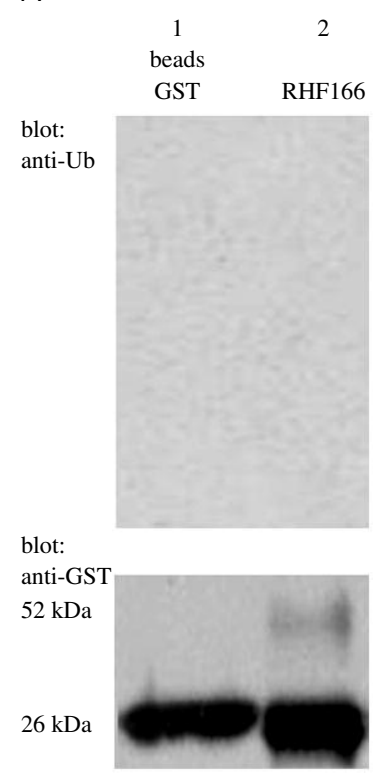

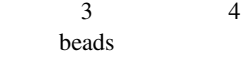

RNF166
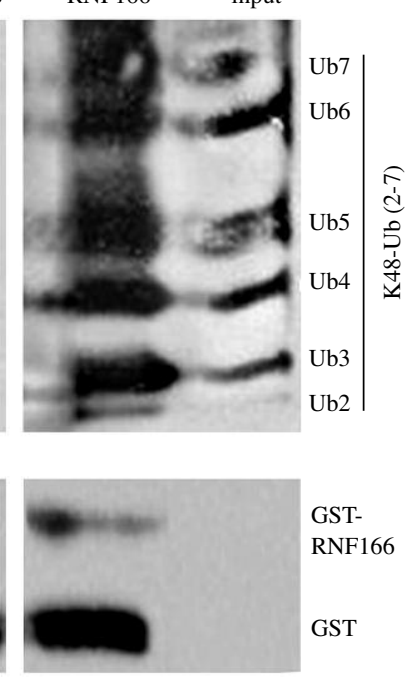

B

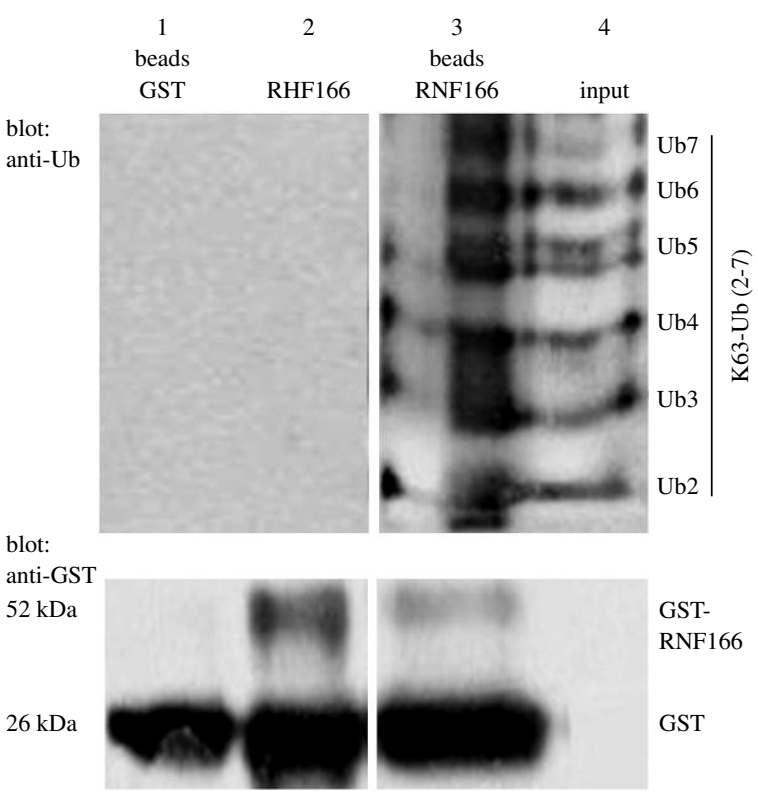

Fig. 4. Polyubiquitin pull-down analysis. A) RNF166 proteins can pull down Lys ${ }^{48-l i n k e d ~ p o l y u b i q u i t i n} 2-7$ chains. B) RNF166 proteins can pull down Lys $^{63}$-linked polyubiquitin ${ }_{2-7}$ chains. Lane 1 and lane 3: wild-type GST (beads) and GST-RNF166 fusion proteins (beads) were isolated on glutathione-Sepharose beads and incubated with Lys ${ }^{48}$-linked polyubiquitin $2-7$ chains $\left(\mathrm{K}^{48}\right.$ $\mathrm{Ub})$ and Lys ${ }^{63}$-linked polyubiquitin ${ }_{2-7}$ chains $\left(\mathrm{K}^{63}-\mathrm{Ub}\right)$ separately. Lane 2: negative controls were GST-RNF166 fusion proteins unincubated with Lys ${ }^{48}$ - or Lys ${ }^{63}$-linked polyubiquitins. Lane 4: positive controls were Lys ${ }^{48}$ - or Lys ${ }^{63}$-linked polyubiquitins inputs alone. Western blots were performed to identify the polyubiquitin-binding ability of RNF166 proteins with anti-ubiquitin antibodies (top panels). GST-RNF166 fusion proteins and wild-type GST were detected with anti-GST antibodies (bottom panels)

through non-proteasome-dependent pathways (mediated by Lys $^{63}$-linked polyubiquitin chains).

\section{Discussion}

The protein subfamily of RING domain E3 ubiquitin ligases, containing four known members: RNF125, RNF114, RNF138 and RNF166, was defined recently based on the protein domain organization [6]. Previous researches have confirmed that RNF125 is a positive regulator of T cells activation, RNF138 can inhibit Wnt signaling pathway by degrade $\mathrm{TCF} / \mathrm{LEF}$ proteins with linking polyubiquitin via UIM domain, and RNF114 is a kind of novel psoriasis susceptibility gene with a critical role in the regulation of immune responses [11-13]. The amino acid similarities between RNF166 and the other three members of this subfamily indicate that RNF166 may be involved in similar physiological activities, especially in cell immune responses, but the functions of RNF166 remained largely unclear yet.

To gain insights into the function of RNF166, we characterized the intact protein and the domain-deletion mutants of RNF166 by flow cytometry respectively. The T cells activation efficiency by flow cytometry analysis showed that intact RNF166 protein with a $N$-terminal GFP tag may effi- ciently upregulate the expression of CD69 (Fig. 3D and Fig. 3 F, panel left 2) compared with wild-type GFP protein transfected as a negative control (Fig. 3D and Fig. 3F, panel left 1 ) in primary T cells and Jurkat T cells, preliminarily confirming that RNF166 was a putative positive regulator of $\mathrm{T}$ cells activation.

To explore the underlying regulating mechanism of RNF166 and study its polyubiquitin-binding properties, recombinant RNF166 protein with a $N$-terminal GST tag was purified from Escherichia coli strain BL21 (DE3) cells on glutathione sepharose beads and was incubated respectively with Lys ${ }^{48}$-linked polyubiquitin ${ }_{2-7}$ chains and Lys ${ }^{63}$ linked polyubiquitin ${ }_{2-7}$ chains (Ubiquitin molecules are polymerised to each other via lysine ${ }^{48}$ residue or lysine ${ }^{63}$ residue). It has been clear that Lys $^{48}$-linked polyubiquitin $2-7$ chains may signal the ubiquitin-proteasome proteolysis pathway sufficiently and mediate degradations of the target substrate proteins which bind these polyubiquitin chains with 26S proteasomes [14]. And Lys ${ }^{63}$-linked polyubiquitin $2-7$ chains have been identified as signals of certain non-proteasome-dependent pathways which involved in several intracellular physiological responses, such as DNA repair, kinase activation, autophagy and translation regulation [15-17]. The polyubiquitin pull-down assay showed that 
RNF166, just like RNF114 and RNF125, can bind both of these two kinds of polyubiquitin chains (Fig. 4). This result indicates that RNF166 may perform the regulating roles in $T$ cells activation by self-degradation via ubiquitin-proteasome pathway and/or cross-talk with certain signaling pathways via non-proteasome-dependent pathways [18].

RING and UIM domains of RNF125 were essential for the regulation of immune responses, but their roles in RNF166 remained unrevealed [5, 6]. The results of flow cytometry analysis in the present study clearly demonstrated that RNF166- $\triangle$ RING showed a potent positive effect (Fig. 3F, panel left 3) contrast with intact RNF166 (Fig. 3F, panel left 2). Similar effect was detected for RNF166- $\Delta$ UIM and other mutants, but not so drastic (Fig. 3F, right four panels). Combining these data with the polyubiquitin-binding ability and E3 enzyme properties of RNF166, we predict that the domains, particularly the RING and UIM domains, participate in the self-ubiquitination of RNF166 protein, just like RNF125 [6]. Thus, though intact RNF166 protein upregulates $\mathrm{T}$ cell activation, its influence was weakened by self-ubiquitination induced by certain domains, such as RING and UIM. And when these relevant domains were deleted, the self-ubiquination of RNF166 was blocked and the activation of $\mathrm{T}$ cells was largely increased.

In addition, previous study has shown that RNF166 may be a putative nucleic acid binding protein [19]. Here, the Lys ${ }^{63}$-linked polyubiquitin chains binding ability (Fig. 4) provided more evidence on the functional diversity of RNF166 and brought it further into focus for investigations into its regulation. Currently, the regulation mechanisms including interaction proteins screening of RNF166 involved in $\mathrm{T}$ cells activation are being addressed in our laboratory.

In conclusion, our study first reported RNF166 as a potential positive regulator in $\mathrm{T}$ cells activation. The data presented in this article provide a new insight into understanding the function of RNF166.

This work was supported by the National Basic Research Program of China (973 Program, 2012CB947600), National Natural Science Foundation of China (90919006 and 31070676), Trans-Century Training Program Foundation for the Talents by the Ministry of Education of China (NCET07-0580) and Spring Sunshine Program by the Ministry of Education of China (Z2009-1-61003).

The authors declare no conflict of interest.

\section{References}

1. Karin M, Ben NY (2000): Phosphorylation meets ubiquitination: the control of NF-[kappa]B activity. Annu Rev Immunol 18: 621-663.

2. Rao N, Lupher ML, Ota S, et al. (2000): The linker phosphorylation site Tyr292 mediates the negative regulatory effect of Cbl on ZAP-70 in T cells. J Immunol 164: 4616-4626.
3. Heissmeyer V, Macian F, Im SH, et al. (2004): Calcineurin imposes $\mathrm{T}$ cell unresponsiveness through targeted proteolysis of signaling proteins. Nat Immunol 5: 255-265.

4. Lu Z, Xu S, Joazeiro C, et al. (2002): The PHD domain of MEKK1 acts as an E3 ubiquitin ligase and mediates ubiquitination and degradation of ERK1/2. Mol Cell 9: 945-956.

5. Jackson PK, Eldridge AG, Freed E, et al. (2000): The lore of the RINGs: substrate recognition and catalysis by ubiquitin ligases. Trends Cell Biol 10: 429-439.

6. Giannini AL, Gao Y, Bijlmakers MJ (2008): T-cell regulator RNF125/TRAC-1 belongs to a novel family of ubiquitin ligases with zinc fingers and a ubiquitin-binding domain. Biochem J 410: 101-111.

7. Chu P, Pardo J, Zhao H, et al. (2003): Systematic identification of regulatory proteins critical for T-cell activation. J Biol 2: 21 .

8. Yamada M, Ohnishi J, Ohkawara B, et al. (2006): NARF, an nemo-like kinase (NLK)-associated ring finger protein regulates the ubiquitylation and degradation of T cell factor/lymphoid enhancer factor (TCF/LEF). J Biol Chem 281: 2074920760.

9. Gerhard DS, Wagner L, Feingold EA, et al. (2004): The status, quality, and expansion of the NIH full-length cDNA project: the Mammalian Gene Collection (MGC). Genome Res 14: 2121-2127.

10. Kimura K, Wakamatsu A, Suzuki Y, et al. (2006): Diversification of transcriptional modulation: large-scale identification and characterization of putative alternative promoters of human genes. Genome Res 16: 55-65.

11. Bijlmakers MJ, Kanneganti SK, Barker JN, et al. (2011): Functional analysis of the RNF114 psoriasis susceptibility gene implicates innate immune responses to double-stranded RNA in disease pathogenesis. Hum Mol Genet 20: 3129-3137.

12. Capon F, Bijlmakers MJ, Wolf N, et al. (2008): Identification of ZNF313/RNF114 as a novel psoriasis susceptibility gene. Hum Mol Genet 17: 1938-1945.

13. Onoufriadis A, Simpson MA, Burden AD, et al. (2012): Identification of rare, disease-associated variants in the promoter region of the RNF114 psoriasis susceptibility gene. J Invest Dermatol 132: 1297-1299.

14. Pickart CM, Fushman D (2004): Polyubiquitin chains: polymeric protein signals. Curr Opin Chem Biol 8: 610-616.

15. Haglund K, Dikic I (2005): Ubiquitylation and cell signaling. EMBO J 24: 3353-3359.

16. Lipford JR, Deshaies RJ (2003): Diverse roles for ubiquitindependent proteolysis in transcriptional activation. Nat Cell Biol 5: 845-850.

17. Mukhopadhyay D, Riezman H (2007): Proteasome-independent functions of ubiquitin in endocytosis and signaling. Science 315: 201-205.

18. Haririnia A, D'Onofrio M, Fushman D (2007): Mapping the interactions between Lys48 and Lys63-linked di-ubiquitins and a ubiquitin-interacting motif of S5a. J Mol Biol 368: 753-766.

19. Royland JE, Kodavanti PR (2008): Gene expression profiles following exposure to a developmental neurotoxicant, Aroclor 1254: pathway analysis for possible mode(s) of action. Toxicol Appl Pharmacol 231: 179-196. 